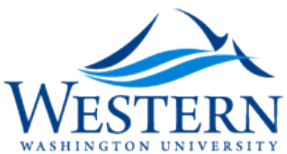

Western Washington University Western CEDAR

7-1985

\title{
On the Regularity of the Critical Point Infinity of Definitizable Operators
}

Branko Ćurgus

Follow this and additional works at: https://cedar.wwu.edu/math_facpubs

\section{Recommended Citation}

Curgus, Branko, "On the Regularity of the Critical Point Infinity of Definitizable Operators" (1985). Mathematics. 75. https://cedar.wwu.edu/math_facpubs/75 
Integral Equations and Operator Theory vol. 8 (1985)
$0378-620 x / 85 / 040462-27 \$ 1.50+0.20 / 0$

(C) 1985 Birkhäuser Verlag, Basel

\begin{abstract}
ON THE REGULARITY OF THE CRITICAL POINT INFINITY OF DEFINITIZABLE OPERATORS
\end{abstract}

Branko Curgus

In this note necessary and sufficient conditions for the regularity of the critical point infinity of a definitizable operator $A$ are given. Using these criteria it is proved that the regularity of the critical point infinity is preserved under some additive perturbations as well as for some operators which are related to $A$. Applications to indefinite sturm-Liouville problems are indicated.

\title{
INTRODUCTION
}

Let $(K,[\ldots])$ be a Krein space (see [3]), $J$ a fundamental symmetry on $K,(\mathrm{x}, \mathrm{y}):=[\mathrm{Jx}, \mathrm{y}](\mathrm{x}, \mathrm{y} \in K)$ and $\|\cdot\|$ the corresponding Hilbert space norm. All topological notions in the Krein space $K$ are understood with respect to the topology generated by the norm II II . This topology does not depend on the special choice of the fundamental symmetry $\mathrm{J}$. For this and other facts about Krein spaces see [3]. We use the common definitions of symmetric, positive, selfadjoint and definitizable operators in Krein spaces (see [3], [19]). In this note all these operators are supposed to be densely defined. A definitizable operator in the Krein space $K$ has a spectral function, possibly with critical points on the real axis, see [3], [9], [19]. The spectral function of the definitizable operator A will be denoted by $E$, and the set of critical points of $E$, which are also called the critical points of $A$, by $c(A)$. The critical point $t \in \mathbb{R}$ of the definitizable operator $A$ is called regular (see [19], [10]) if there exists an open neighbourhood $\Delta_{0} \cong \mathbb{R}$ of $t, \Delta_{0} \cap c(A)=\{t\}$, such that the projectors $E(\Delta), \bar{\Delta} \cong \Delta_{0} \backslash\{t\}$ are uniformly 
bounded. Here $\overline{\mathbb{R}}=\mathbb{R} \cup\{\infty\}$ is regarded as the one point compactification of $\mathbb{R}$. The critical points of $A$ which are not regular are called singular critical points of $A$; we denote the set of all singular critical points of $A$ by $c_{S}(A)$.

In this note we give criteria for the regularity of the critical point $\infty$ of the definitizable operator A. E.g., we show that infinity is not a singular critical point of $A$ if and only if in the Krein space $K$ there exists a positive, bounded and boundedly invertible operator $W$ such that $W D(A) \leqq D(A)$. Further, we use these criteria in order to prove that the regularity of the critical point $\infty$ is preserved under some additive perturbations as well as for some operators which are related to $A$.

We mention that the main criteria for the regularity of the critical point $\infty$ given in Theorems 2.5 and 3.2 are inspired by a construction of Beals (see [2]), see Remark 3.7. In [5] we use the above results in order to study selfadjoint, ordinary differential operators with an indefinite weight function.

\section{PRELIMINARIES}

1.1. For the convenience of the reader we recall the following results.

PROPOSITION 1.1. Let $\left(H_{1},(\ldots)_{1}\right)$ and $\left(H_{2},(\ldots)_{2}\right)$ be Hilbert spaces such that $H_{1}$ is dense in $\left(H_{2},(.,)_{2}\right)$ and for some constant $\mathrm{k}>0$ holds $\|\mathrm{x}\|_{1} \geqq \mathrm{k}\|\mathrm{x}\|_{2}\left(\mathrm{x} \in H_{1}\right)$. Then there exists a positive selfadjoint operator in $\mathrm{H}_{2}$ with domain $H_{1}$. If $\mathrm{P}$ is any selfadjoint operator in $\mathrm{H}_{2}$ with $D(P)=H_{1}$, then the norm $\|\cdot\|_{1}$ is equivalent to the graph norm

$$
\mathrm{x} \mapsto\left(\|\mathrm{x}\|_{2}^{2}+\|\mathrm{Px}\|_{2}^{2}\right)^{1 / 2} \quad\left(\mathrm{x} \in \mathrm{D}(\mathrm{P})=H_{1}\right) \cdot(1.1)
$$

The first statement follows from Theorem 2.23 in [13]. To prove the second statement we observe that the inequality 


$$
\left(\|x\|_{2}^{2}+\|P x\|_{2}^{2}\right)^{1 / 2} \geqq\|x\|_{2} \quad\left(x \in H_{1}\right)
$$

holds, we form a sum of the norm (1.1) and $\|\cdot\|_{1}$, and apply the closed graph theorem.

We note that in the case $0 \in \rho(P)$ the norm (1.1) is equivalent to the norm $\mathrm{x} \rightarrow\|P x\|_{2}\left(x \in D(P)=H_{1}\right)$.

THEOREM 1.2. ("Heinz inequality", see [16].) Let $P$ and $\mathrm{P}_{1}$ be positive selfadjoint operators defined in the Hirbert spaces $(H,(.)$,$) and \left(H_{1},(.,)_{1}\right)$, respectively. If $\mathrm{T}: H \rightarrow H_{1}$ is a bounded operator with the norm $\mathrm{M}$ such that $T D(P) \cong D\left(P_{1}\right)$ and

$$
\left\|P_{1} T x\right\|_{1} \leqq M_{1}\|P x\| \quad(x \in D(P)),
$$

then, for $0 \leqq \alpha \leqq 1$, we have $\mathrm{T} D\left(\mathrm{P}^{\alpha}\right) \leqq D\left(\mathrm{P}_{1}^{\alpha}\right)$ and

$$
\left\|\mathrm{P}_{1}^{\alpha} \mathrm{Tx}\right\|_{1} \leqq \mathrm{M}^{1-\alpha} \mathrm{M}_{1}^{\alpha}\left\|\mathrm{P}^{\alpha} \mathrm{x}\right\| \quad\left(\mathrm{x} \in \mathrm{D}\left(\mathrm{P}^{\alpha}\right)\right) \text {. }
$$

The following corollary is a consequence of Theorem 1.2 and Proposition 1.1.

COROLLARY 1.3. If $\mathrm{P}_{1}$ and $\mathrm{P}_{2}$ are positive selfadjoint operators in a Filbert space $H$ and $D\left(\mathrm{P}_{1}\right)=D\left(\mathrm{P}_{2}\right)$, then

$$
D\left(P_{1}^{\alpha}\right)=D\left(P_{2}^{\alpha}\right) \quad(0 \leqq \alpha \leqq 1)
$$

and the corresponding graph norms on $D\left(P_{j}^{\alpha}\right)(j=1,2)$ are equivalent.

In a special case the equality in (1.2) holds for arbitrary nonnegative $\alpha$. Namely, let $s$ be a selfadjoint operator in the Hilbert space $H$ which is bounded from below with a lower bound $\gamma$. Then for $a \leqq \gamma$ it holds

$$
D\left((s-a I)^{\alpha}\right)=D\left(|s|^{\alpha}\right) \quad(\alpha \in[0,+\infty)) \text {. }
$$

This follows easily using the characterization of the elements of the domains in (1.3) by means of the spectral function of $\mathrm{S}$.

1.2. Let $A$ be a selfadjoint operator in the Krein space $(K,[, \ldots])$. According to Proposition 1.1 the topology 
on $D(A)$ generated by the graph norm with respect to the operator JA does not depend on the special choice of $J$. The set $D(A)$ equipped with this topology will be denoted by $D(A) \wedge$. For a selfadjoint operator $S$ in the Hilbert space $(H,(.)$,$) by D[S]$ we denote the completion of $D(S)$ with respect to the norm $\|(|S|+I)^{1 / 2}$. $\|$. The linear space $D[S]$ with the topology defined by the norm $\|(|S|+I)^{1 / 2}$. $\|$ is denoted by $D[S]^{\sim}$. Since the operator $(|S|+I)^{1 / 2}$ is boundedly invertible ${ }^{1)}$ we have

$$
\|x\| \leqq\left\|(|S|+I)^{-1 / 2}\right\|\left\|(|S|+I)^{1 / 2} x\right\| \quad(x \in D(S)),
$$

and this implies $D[S] \subseteq H$. It holds $D[S]=D\left((|S|+I)^{1 / 2}\right)$ and, by $(1.2), D[S]=D\left(|S|^{1 / 2}\right)$.

According to Corollary $1.3 \mathrm{D}[\mathrm{JA}]^{\sim}$, defined in the

Hilbert space $(K,(.)$,$) , does not depend on the special choice$ of $\mathrm{J}$.

REMARK 1.4. Proposition 1.1 and Corollary 1.3 imply that the following equalities hold true

$$
\begin{aligned}
& D[J A]^{\sim}=D[|J A|+I]^{\sim}=D\left(J(|J A|+I)^{1 / 2}\right)^{\wedge}, \\
& D(A)^{\wedge}=D(J(|J A|+I))^{\wedge}=D\left[(J A)^{2}\right]^{\sim} .
\end{aligned}
$$

REMARK 1.5. Let $A$ be a selfadjoint operator in the Krein space $(K,[, \ldots])$, such that the inequality $[A x, x] \geqq \gamma\|x\|^{2}(x \in D(A))$ is satisfied for some real constant $\gamma$ depending on the fundamental symmetry $J, i . e$. the sesquilinear form $[A .]:, D(A) \times D(A) \rightarrow \mathbb{C}$ is bounded from below in $(K,(\ldots))$. Then the operator JA is selfadjoint and bounded from below in the Hilbert space $(K,(\ldots))$. Now Remark 1.4 and Corollary 1.3 imply that $D[J A]$ is the domain of the closure of the sesquilinear form $[A, \ldots]$ (see $[23$, p. 122]).

1) An operator $A$ is said to be boundedly invertible if $0 \in \rho(A)$. 
REMARK 1.6. If the selfadjoint operator $S$ is given by an ordinary $2 n$-th order differential expression with boundary conditions the set $D[S]$ is determined by the essential boundary conditions (see $\left[14,10^{\circ}\right],[4$, Theorem 2.4]).

REMARK 1.7. Let $S$ be a boundedly invertible selfadjoint operator in the Hilbert space $(H,(, \ldots))$. The topology on $D[S]^{\sim}$ is given by the norm $\left\||S|^{1 / 2}.\right\|$. The inequality

$$
|(s x, y)| \leqq\left\||s|^{1 / 2} x\right\|\left\||s|^{1 / 2} y\right\| \quad(x, y \in D(s))
$$

implies that the scalar product $(S .,$.$) can be extended by con-$ tinuity onto $D[S]$. Then $(D[S],(S .,)$.$) is a Krein space.$ The norm topology on this krein space is defined by $\left\||\mathrm{s}|^{1 / 2}.\right\|$, hence it coincides with the topology on $D[S]^{\sim}$. This Krein space is a Pontrjagin space of index $K$ (see [19]) if and only if the negative spectrum of $S$ consists of finitely many eigenvalues of total multiplicity $k$. In this case the operator $s$ is bounded from below, say $s \geqq \gamma$, and Corollary 1.3 yields that the norm $\|(S-a I)^{1 / 2}$. $\|$, for $a<\gamma$, generates the norm topology of the Pontrjagin space $(D[s],(s .,)$.$) .$

1.3. In this subsection we consider a selfadjoint operator $A$ in the Krein space $(K,[.,]$.$) such that zero is not$ an eigenvalue of $A$ and put $P=J A$. Then the following equivalences hold true.

(a) If the operator PJP is densely defined, then it is selfadjoint in the Hilbert space $(K,(.)$,$) if and only if$ $\mathrm{P}^{-1} \pm$ ai $\mathrm{PJ}$ are boundedly invertible operators for some (and hence for all) a $\in \mathbb{R}$.

(b) The resolvent set $\rho\left(\mathrm{JP}^{2}\right)$ is not empty if and only if the operator $\mathrm{P}^{-1}+$ ai JP is boundedly invertible for some (and hence for all) a $\in \mathbb{R}$.

We mention that the operators $\mathrm{P}^{-1} \pm$ ai $\mathrm{PJ}$ are defined on PD (PJP). In order to prove (a) we suppose first that PJP is a selfadjoint operator. Then for every a $\in \mathbb{R}$ the operator I - ai PJP is boundedly invertible. The operator $P(I-a i \text { PJP })^{-1}$ is a composition of the bounded operator 
$(I-a i \text { PJP })^{-1}$, which maps $K$ onto $D(P J P) \leqq D(P)$, and the closed operator $P$. Hence, $P(I-\text { ai PJP })^{-1}$ is an everywhere defined closed operator and therefore bounded. It is easy to see that $P(I-a i \text { PJP })^{-1}$ is an inverse of $\mathrm{P}^{-1}-$ ai PJ . Conversely, let us suppose that the operators $\mathrm{P}^{-1} \pm$ ai $\mathrm{PJ}$ are boundedly invertible for some $a \in \mathbb{R}$. Then $\mathrm{P}^{-1}\left(\mathrm{P}^{-1} \pm \text { ai } \mathrm{PJ}\right)^{-1}$ are everywhere defined closed operators and hence bounded. These operators are the inverses of the operators $I \pm$ ai PJP . Therefore, the symmetric operator PJP is selfadjoint in the Hilbert space $(K,(.)$,$) .$

The proof of (b) uses the same ideas as the proof of (a).

LEMMA 1.8. Let A be a definitizable operator in the Krein space $K$ such that zero is not an eigenvalue of $A$ and put $\mathrm{P}=\mathrm{JA}$. If $\mathbb{R} \cap \rho(\mathrm{P}) \neq \varnothing$, then for any positive integer $\mathrm{m}$ the operator $\mathrm{JP}^{2^{\mathrm{m}}}$ has a nonempty resolvent set.

PROOF. We shall prove the lemma for $m=1$ first. Let $1 / b$ be a real number in $\rho(P)$. The nonreal spectrum of $A$ consists of a finite number of points. Therefore we can choose a real $a \neq 0$ such that $-a i, a i, i / a b \in \rho(A)$.

1. We have chosen the number a such that the operator

$$
(A-a i I)(A+a i I)=A^{2}+a^{2} I
$$

is boundedly invertible, i.e. $-a^{2} \in \rho\left(A^{2}\right)$. Consequently, the symmetric operator $A^{2}+a^{2} I$ is selfadjoint in the krein space $K$. Hence, $A^{2}$ is selfadjoint in the Krein space $K$ and $\mathrm{PJP}=\mathrm{JA}^{2}$ is selfadjoint in the Hilbert space $(K,(\ldots))$. According to the equivalence (a) the operator $\mathrm{P}^{-1}-$ ai PJ is boundedly invertible. Now we prove that $\mathrm{P}^{-1}-\mathrm{ai} P J$ is a densely defined operator. The operator $\mathrm{PJ}=J A J$ is invertible and definitizable in the Krein space $K$. Indeed, PJ is a selfadjoint operator in $(K,[.]$,$) , it has the same defini-$ tizing polynomial as $A$ and $\rho(J A J)=\rho(A) \neq \varnothing$. Therefore, $D(P J) \cap R(P J)=D\left(P^{-1}-a i\right.$ PJ $)$ is dense in $K$ and $P^{-1}-a i$ PJ is a densely defined and boundedly invertible operator. 
2. According to the choice of $a$ and $b$ the operator

$$
\left(J P+\frac{i}{a b} I\right)\left(b P^{-1}-I\right)=b J-J P+\frac{i}{a} P^{-1}-\frac{i}{a b} I
$$

is boundedly invertible and hence closed. Thus, $\frac{i}{a} P^{-1}-J P$ and also $\mathrm{P}^{-1}+\mathrm{ai} \mathrm{JP}$ are closed operators. Comparing the domains we see that

$$
\mathrm{P}^{-1}(I+a i \mathrm{PJP}) \subseteq \mathrm{P}^{-1}+\mathrm{ai} \mathrm{JP}
$$

Consequently

$$
R\left(P^{-1}+a i J P\right) \supseteqq R\left(P^{-1}(I+a i P J P)\right)=D(P) .
$$

Hence, we see that $\mathrm{P}^{-1}+\mathrm{ai} \mathrm{JP}$ is a densely defined, closed operator with a dense range.

3. It holds true

$$
\mathrm{P}^{-1}+\mathrm{ai} J \mathrm{P} \cong\left(\mathrm{P}^{-1}-\mathrm{ai} \mathrm{PJ}\right)^{*}
$$

where the asterisk * denotes operator adjoints in $(K,(., 1))$. According to the first step of this proof the operator on the right-hand side of $(1.4)$ is boundedly invertible and we have

$$
\left(\mathrm{P}^{-1}+a i J P\right)^{-1} \subseteq\left(\mathrm{P}^{-1}-\mathrm{ai} \mathrm{PJ}\right)^{*-1}
$$

According to the second step of this proof the operator on the left-hand side of (1.5) is densely defined and closed. Therefore, in (1.5) (and hence in (1.4)) we have equality and $\mathrm{P}^{-1}+\mathrm{ai} J \mathrm{P}$ is a boundedly invertible operator.

The equivalence (b) yields $\rho\left(J P^{2}\right) \neq \varnothing$. Hence the lemma is proved for the case $m=1$. If $m>1$, we apply the already proved result $\mathrm{m}$ times. The lemma is proved.

In a similar way we show that for a selfadjoint invertible operator $P$ in the Hilbert space $(K,(\ldots))$ such that $\rho\left(\mathrm{JP}^{2}\right) \neq \varnothing$ the operator $\mathrm{PJP}$ is selfadjoint in $(K,(\ldots))$. This result improves Lemma 3.1 from [18] where only the essential selfadjointness of the operator PJP is proved. 
In order to prove the stated result we observe that $\mathrm{P}^{-1} \pm$ ai $\mathrm{PJ}$ are closed operators with dense ranges. Indeed, these operators are closed as the operators

$$
\left(P^{-1}+I\right)(a i P J \pm I)=a i J+a i P J \pm P^{-1} \pm I
$$

are closed, and the inclusions

$$
\mathrm{P}^{-1} \pm \text { ai } \mathrm{PJ} \supseteqq \mathrm{P}^{-1}\left(I \pm a i \mathrm{P}^{2} \mathrm{~J}\right)
$$

imply that the operators $\mathrm{P}^{-1} \pm$ ai $\mathrm{PJ}$ have dense ranges.

$$
\text { It holds true }
$$

$$
\mathrm{P}^{-1} \pm \mathrm{ai} \mathrm{PJ} \cong\left(\mathrm{P}^{-1} \mp \mathrm{ai} \mathrm{JP}\right)^{*}
$$

According to the equivalence (b) the operators on the right-hand side of $(1.6)$ are boundedly invertible and we have

$$
\left(\mathrm{P}^{-1} \pm \text { ai } \mathrm{PJ}\right)^{-1} \cong\left(\mathrm{P}^{-1} \mp \text { ai JP }\right)^{*-1} \text {. }
$$

According to the preceding observation the operators on the left-hand side of (1.7) are densely defined and closed. Therefore, in (1.7) (and hence in (1.6)) we have equality and $\mathrm{P}^{-1} \pm \mathrm{ai}$ PJ are boundedly invertible operators. The equivalence (a) yields that PJP is a selfadjoint operator in $(k,(.,)$.

\section{POSITIVE OPERATORS}

2.1. In this section we consider a positive, boundedly invertible operator $A$ in the Krein space $(K,[, \ldots])$. Then we have

$$
[A x, x]=(J A x, x) \geq\left\|(J A)^{-1}\right\|^{-1}\|x\|^{2}=\left\|A^{-1}\right\|\|x\|^{2}(x \in D(A)),
$$
and

$$
(x, y)_{A}:=[A x, y] \quad(x, y \in D(A))
$$


defines a positive definite scalar product on $D(A)$. The corresponding norm $\left\|(\mathrm{JA})^{1 / 2} \cdot\right\|$ is denoted by $\|\cdot\|_{A}$. As in Remark 1.7 the scalar product $(.,)_{A}$ can be extended by continuity onto $D[J A]$ and $\left(D[J A],(.,)_{A}\right)$ is a Hilbert space. REMARK 2.1. The operator $A^{-1} \mid D[J A]$ maps $D[J A]$ into $D(A) \subseteq D[J A]$ and it is a selfadjoint, bounded operator in the Hilbert space $\left(D[J A],(\ldots)_{A}\right)$. Indeed, the selfadjointness follows from the relation

$$
\begin{aligned}
& \left(A^{-1} x, y\right)_{A}=\left[A A^{-1} x, y\right]=\left[A^{-1} A x, y\right] \\
& =\left[A x, A^{-1} y\right]=\left(x, A^{-1} y\right)_{A} \quad(x, y \in D(A)),
\end{aligned}
$$

and the boundedness from the relation

$$
\begin{aligned}
& \left|\left(A^{-1} x, x\right)_{A}\right|=|[x, x]| \leqq(x, x) \\
& \leqq\left\|A^{-1}\right\|[A x, x]=\left\|A^{-1}\right\|(x, x)_{A} \quad(x \in D(A)) .
\end{aligned}
$$

The next lemma is a simple consequence of Krein-ReidLax theorem about symmetrizable operators (see [15], [21], [20]). LEMMA 2.2. Let $\mathrm{S}$ and $\mathrm{K}$ be bounded operators in the Hilbert space $(H,(.)$,$) such that S$ and $S K$ are selfadjoint. Then we have

$$
|(S K x, x)| \leqq\|K\|(|S| x, x) \quad(x \in H) .
$$

PROOF. The operator $\operatorname{sgn}(\mathrm{S}) \mathrm{K}$ is bounded in $H$ and $\|\operatorname{sgn}(S) K\| \leqq\|K\|$. The operator $|S|$ is positive and $|\mathrm{S}| \operatorname{sgn}(\mathrm{S}) \mathrm{K}=\mathrm{SK}$ is a selfadjoint operator in $H$. Therefore all the assumptions of Theorem 2.1 in [21] are satisfied. Hence

$$
\begin{aligned}
& |(S K x, x)|=|(|S| \operatorname{sgn}(S) K x, x)| \\
& \leqq\|K\|(|S| x, x) \quad(x \in H) .
\end{aligned}
$$

The lemma is proved.

LEMMA 2.3. Let $B$ be a positive, boundedly invertible operator in the Krein space $(K,[\ldots])$. Assume that there 
exists a positive, boundedly invertible operator $\mathrm{W}$ in the Krein space $(K,[. .]$.$) such that$

$$
D[J B] \cong D(W), W D[J B] \cong D[J B]
$$

and such that $\left.W\right|_{D[J B]}$ is a bounded operator in $D[J B]^{\sim}$. Then, on $D[\mathrm{JB}]$ the norm generated by the positive definite scalar product

$$
\langle\mathrm{x}, \mathrm{y}\rangle_{\mathrm{B}}:=\left(\left|\mathrm{B}^{-1}\right|_{D[\mathrm{JB}]} \mid \mathrm{x}, \mathrm{y}\right)_{\mathrm{B}} \quad(\mathrm{x}, \mathrm{y} \in \mathrm{O}[\mathrm{JB}])
$$

is equivalent to the Hilbert space norm $\|\cdot\|$ of $K$. The operator $\mathrm{W}$ is bounded in $K$.

PROOF. The operator $\left.B^{-1}\right|_{D[J B]}$ is selfadjoint and bounded in the Hilbert space $\left(D[J B],(., .)_{B}\right) ;\left|B^{-1}\right| O[J B]$ denotes its absolute value in this Hilbert space. For $x \in D[J B]$ we have

$$
\begin{aligned}
& \|x\|^{2}=(x, x) \leqq\left\|W^{-1}\right\|[W x, x]=\left\|W^{-1}\right\|\left(B^{-1} W x, x\right)_{B} \\
& \left.\leqq\left\|W^{-1}\right\|\left\|\left.W\right|_{D[J B]}\right\| \|\left. B^{-1}\right|_{D[J B]} \mid x, x\right)_{B} \\
& =\left\|W^{-1}\right\|\left\|\left.W\right|_{D[J B]}\right\|\langle x, x\rangle_{B} .
\end{aligned}
$$

The last inequality in (2.2) is a consequence of Lemma 2.2 applied to the bounded operators $\left.B^{-1}\right|_{D[J B]}$ and $\left.W\right|_{D[J B]}$ in the Hilbert space $\left(D[J B],(., .)_{B}\right)$. Here we use the fact that the operator $\left(B^{-1} I_{D[J B]}\right)\left(W I_{D[J B]}\right)$ is positive in $\left(D[J B],(. .)_{B}\right)$ (see the first line in (2.2)). Further, for $x \in D[J B]$ we have

$$
\begin{aligned}
\langle\mathrm{x}, \mathrm{x}\rangle_{\mathrm{B}} & =\sup \left\{\left|\langle\mathrm{x}, \mathrm{y}\rangle_{\mathrm{B}}\right|^{2}:\langle\mathrm{y}, \mathrm{y}\rangle_{\mathrm{B}} \leqq 1\right\} \\
& =\sup \left\{\left|\left(\left|\mathrm{B}^{-1}\right|_{D[J \mathrm{~B}]} \mid \mathrm{x}, \mathrm{y}\right)_{\mathrm{B}}\right|^{2}:\langle\mathrm{y}, \mathrm{y}\rangle_{\mathrm{B}} \leqq 1\right\} \\
& =\sup \left\{\left|\left(\mathrm{B}^{-1} \mathrm{x}, \mathrm{y}\right)_{\mathrm{B}}\right|^{2}:\langle\mathrm{y}, \mathrm{y}\rangle_{\mathrm{B}} \leqq 1\right\} \\
& =\sup \left\{|[\mathrm{x}, \mathrm{y}]|^{2}:\langle\mathrm{y}, \mathrm{y}\rangle_{\mathrm{B}} \leqq 1\right\}
\end{aligned}
$$




$$
\begin{aligned}
& \leqq \sup \left\{|[x, y]|^{2}:\|y\|^{2} \leqq\left\|w^{-1}\right\|\|w\|_{D[J B]} \|\right\} \\
& =\left\|w^{-1}\right\|\|w\|_{D[J B]}\|\| x \|^{2} .
\end{aligned}
$$

By (2.2) and (2.3) the scalar products $<\ldots,\rangle_{B}$ and $(\ldots$, generate equivalent norms on $D[J B]$. The operator JB is positive in the Hilbert space $(K,(.)$,$) and from (2.2)$ and (2.3) it follows that $\mathrm{JW}$ is a bounded operator in $(K,(\ldots))$. Here we have used the fact that $D(B)$ is a dense set in $K$. Then the operator $W$ is also bounded. This completes the proof of the lemma.

We note that in [2] and [12], in order to prove halfrange completeness, the equivalence of the norms in Lemma 2.4 was shown by other methods for the special case of sturmLiouville operators with an indefinite weight function.

In the following, if $J$ is a fundamental symmetry we put $\mathrm{P}_{ \pm}:=\frac{1}{2}(I \pm \mathrm{J}), K_{ \pm}:=\mathrm{P}_{ \pm} K$.

The operator $A$ in the Krein space $K$ is called fundamentally reducible if there exists a fundamental symmetry $J$ such that for every $x \in D(A)$ we have $P_{+} x, P_{-} x \in D(A)$ and $\mathrm{AP}_{ \pm} \mathrm{x} \in K_{ \pm}$.

The following characterization of fundamental reducibility is contained in [6].

LEMMA 2.4. The following statements are equivalent.

(i) The operator $\mathrm{A}$ is fundamentally reducible.

(ii) There exists a fundamental symmetry $\mathrm{J}$ such that $\mathrm{AP}_{+} \supseteq \mathrm{P}_{+} \mathrm{A}$ and $\mathrm{AP}_{-} \supseteq \mathrm{P}_{-} \mathrm{A}$ hold.

(iii) There exists a fundamental symmetry $\mathrm{J}$ such that $\mathrm{AJ}=\mathrm{JA}$ holds.

The operator $A$ in the Hilbert space $(H,(.)$,$) is$ said to be similar to a selfadjoint operator in $(H,(.)$,$) if$ there exists a scalar product $(\ldots,)^{\prime}$ on $H$ such that $(\ldots,)^{\prime}$ and $(.,$.$) generate on H$ equivalent norms and $A$ is selfadjoint in the Hilbert space $\left(H,(.,)^{\prime}\right)$. The following 
theorem is the main result of this section.

THEOREM 2.5. Let A be a positive, boundedly invertible operator in the Krein space $(K,[\ldots])$. The following statements are equivalent.

(i) $\mathrm{A}$ is fundamentally reducible.

(ii) In the Krein space (K,[.,.]) there exists a positive, boundedly invertible operator $\mathrm{w}$ such that

$$
D(A) \subseteq D(W), W D(A) \subseteq D(A)
$$

and $\mathrm{WI} D(\mathrm{~A})$ is a bounded operator in $D(\mathrm{~A}) \hat{}$.

(iii) In the Krein space (K,[...]) there exists a positive, bounded and boundedly invertible operator $W$ such that (2.4) holds.

(iv) In the Krein space $(K,[.]$.$) there exists a$ positive, boundedly invertible operator $\mathrm{W}$ such that

$$
D[J A] \leqq D(W), W D[J A] \leqq D[J A],
$$

and $W I_{D[J A]}$ is a bounded operator in $D[J A]^{\sim}$.

(v) The positive definite scalar products $<.,{ }_{\mathrm{A}}$

and (...) generate equivalent norms on $D[J A]$.

(vi) A is similar to a selfadjoint operator in the Hizbert space $(K,(\ldots))$.

(vii) Infinity is not a singular critical point of $A$.

PROOF. (i) $\Rightarrow$ (ii): Let $A$ be fundamentally reducible.

Then by Lemma 2.4 there exists a fundamental symmetry $J_{0}$ which commutes with $A, A J_{0}=J_{0} A$. It follows that $J_{0} D(A) \subseteq D(A)$, and $J_{0}$ is bounded with respect to the norm $\|\cdot\|_{A J_{0}}$. According to Proposition 1.1 the norm $\|\cdot\|_{A_{0} J_{0}}$ generates on $D(A)$ the topology of $D(A) \hat{}$. Hence, $J_{O} I_{D(A)}$ is a bounded operator in $D(A)$ and we can take $W=J_{0}$ in (ii).

(ii) $\Rightarrow$ (iii): In order to prove this implication we apply Lemma 2.3 to the operators $B:=A J A$ and $W$ from (ii). The operator $A J A$ is positive and boundedly invertible 
in the Krein space $K$. According to Remark 1.4 we have $D\left[(J A)^{2}\right]=D[J(A J A)]=D(A)$. Hence all the assumptions of Lemma 2.3 are fulfilled and according to this lemma the operator $W$ is bounded in $K$.

(iii) $\Rightarrow$ (iv): The operator $\mathrm{WA}^{-1}$ is bounded in $K$. Since $W D(A) \subseteq D(A)$, the operator $A W A^{-1}$ is everywhere defined and closed, and therefore bounded in $K$. The operator WAWA $^{-1}$ is also bounded in $K$, i.e.

$$
\| \text { WAWA }^{-1} x\left\|\leqq c_{3}\right\| x \| \quad(x \in K)
$$

with some $c_{3}>0$. This inequality is equivalent to

$$
\| \text { WAWx }\left\|\leqq c_{3}\right\| A x \| \quad(x \in D(A)),
$$

and also to

$$
\|J W A W x\| \leqq c_{3}\|J A x\| \quad(x \in D(A)) \text {. }
$$

The operator $c_{3}$ JA is positive and selfadjoint in $(K,(., \ldots))$. The operator JWAW is positive and boundedly invertible, and hence selfadjoint in $(K,(\ldots))$. Furthermore, $D($ JWAW $) \supseteqq D(A)=D\left(c_{3} J A\right)$. BY Theorem 1.2 it follows that

$$
(J W A W x, x) \leqq c_{3}(J A x, x) \quad(x \in D(A)),
$$

or, equivalently,

$$
[A W x, W x] \leqq C_{3}[A x, x] \quad(x \in D(A))
$$

This shows that the operator $W^{\prime} I_{(A)}$ is bounded with respect to the norm $\|\cdot\|_{A}$ on $D(A)$. It remains to show that $W D[J A] \subseteq D[J A]$. Let $\left(x_{n}\right)$ be a sequence of elements of $D(A)$ and $x_{n} \rightarrow x$ in $\|\cdot\|_{A}$. Then $x \in D[J A], x_{n} \rightarrow x$ in $\|\cdot\|$ and, since $W$ is bounded, $W x_{n} \rightarrow W x$ in $\|\cdot\|$. since $\left.W\right|_{D(A)}$ is bounded with respect to $\|\cdot\|_{A}$ on $D(A)$, the sequence $\left(W x_{n}\right)$ is a $\|\cdot\|_{A}$-Cauchy sequence and consequently convergent in $\left(D[J A],\|\cdot\|_{A}\right)$, i.e. $W x_{n} \rightarrow y_{0}$ in $\|\cdot\|_{A}, y_{0} \in D[J A]$. 
It follows $W_{n} \rightarrow y_{0}$ in $\|\cdot\|$. Hence $y_{0}=W x \in D[J A]$. This proves $W D[J A] \leqq D[J A]$.

(iv) $\Rightarrow(v)$ : This implication is a consequence of Lemma 2.3.

$$
\text { (v) } \Rightarrow \text { (vi): Assume that the scalar products }<., .\rangle_{A}
$$

and (.,.) generate equivalent norms on $D[J A]$. Since $D[\mathrm{JA}]$ is dense in the Hilbert space $(K,(\ldots))$ the scalar product $<.,\rangle_{A}$ can be extended onto $K$ by continuity. The extended scalar product will also be denoted by $\langle.,\rangle_{A}$. In Remark 2.1 it was shown that the operator $\left.A^{-1}\right|_{D[J A]}$ is selfadjoint in the Hilbert space $\left(D[J A],(.,)_{A}\right)$. In the same way one proves that the operator $A^{-1}$ is selfadjoint in the Hilbert space $\left(K,\langle\ldots,\rangle_{A}\right)$. The scalar products $\langle\ldots\rangle_{A}$ and $(\ldots)$ generate equivalent norms on $K$ and hence $A^{-1}$ is similar to a selfadjoint operator in the Hilbert space $(K,(\ldots)$,$) . Con-$ sequently, $A$ is also similar to a selfadjoint operator in $(K,(.)$,$) and (vi) is proved.$

The implication (vi) $\Rightarrow$ (vii) is obvious. The equivalence (i) $\Leftrightarrow$ (vii) is well-known (see [1]). The theorem is proved.

In [12] one can find an example of an operator $A$ for which the norms in (v) are not equivalent. According to Theorem 2.5 this means $\infty \in \mathrm{c}_{\mathrm{s}}(\mathrm{A})$. Earlier examples of positive operators in Krein spaces for which $\infty \in \mathrm{c}_{\mathbf{s}}(A)$ were given in [17], [7] and [1].

COROLLARY 2.6. Let $\mathrm{A}$ and $\mathrm{B}$ be positive, boundedly invertible operators in the Krein space $K$ and suppose that $D(A)=D(B)$. Then $\infty \notin \mathrm{c}_{\mathrm{S}}(\mathrm{A})$ if and only if $\infty \notin \mathrm{C}_{\mathrm{S}}(\mathrm{B})$. PROOF. This assertion is an easy consequence of the equivalence (iii) $\Leftrightarrow$ (vii) in Theorem 2.5 and the assumption $D(A)=D(B)$.

COROLLARY 2.7. Let A be as in Theorem 2.5. Then A is fundamentally reducible if and only if there exists a fundamental symmetry $\mathrm{J}$ such that the inclusion $\mathrm{P}_{+} D(\mathrm{~A}) \cong D(\mathrm{~A})$ holds. 
PROOF. The operator $\mathrm{J}=\mathrm{P}_{+}-\mathrm{P}_{-}$is positive, bounded and boundedly invertible in the Krein space $K$ and we have $J D(A) \subseteq D(A)$. Thus the implication (iii) $\Rightarrow$ (i) from Theorem 2.5 implies the "if" part of the corollary. The converse statement is obvious.

2.2. IEMMA 2.8. Let $\mathrm{A}$ be a positive, boundedly invertible operator in the Krein space $K$, and let $\mathrm{m}$ be an integer. put $\mathrm{P}=\mathrm{JA}$. Then $\infty \notin \mathrm{c}_{\mathrm{s}}(\mathrm{A})$ if and only if $\infty \notin \mathrm{c}_{\mathrm{s}}\left(\mathrm{JP}^{2^{\mathrm{m}}}\right)$. PROOF. Suppose first that $m=1$. Then the operator $\mathrm{JP}^{2}=\mathrm{AJA}$ is positive and boundedly invertible in the Krein space $K$. In Remark 1.4 we have noted that $D(A) \hat{~}=D[J(A J A)]^{\sim}$. Therefore the operator $W$ in Theorem 2.5 (ii) with respect to the operator $A$ has the same properties as $W$ in Theorem 2.5 (iv) with respect to the operator AJA. Hence it follows that $\infty \notin \mathrm{C}_{\mathrm{S}}(\mathrm{A})$ if and only if $\infty \notin \mathrm{C}_{\mathrm{S}}\left(\mathrm{JP}^{2}\right)$. Further we can suppose that $m \neq 0$. Then the lemma follows if we apply the already proved part of Lemma $2.8 \mathrm{~m}$ times. In the case $m>0$ we start with the operator $A$, and in the case $\mathrm{m}<0$ we start with $\mathrm{JP}^{2^{\mathrm{m}}}$.

THEOREM 2.9. Let $\mathrm{A}$ be a positive, boundedly invertible operator in the Krein space $K$ and let $\mu \in(0,+\infty)$. Put $\mathrm{P}=\mathrm{JA}$. Then $\infty \notin \mathrm{C}_{\mathbf{s}}(\mathrm{A})$ if and only if $\infty \notin \mathrm{c}_{\mathbf{S}}\left(\mathrm{JP}^{\mu}\right)$. PROOF. Suppose that $\infty \notin \mathrm{C}_{\mathrm{S}}(\mathrm{A})$. The implication (vii) $\Rightarrow$ (ii) in Theorem 2.5 yields the existence of a positive, boundedly invertible operator $w$ such that

$$
D(A) \subseteq D(W), W D(A) \leqq D(A)
$$

and the operator $W I_{D(A)}$ is bounded with respect to the norm $\|\left(P^{2}+I\right)^{1 / 2}$. $\|$ on $D(A)$. Consequently, the operator $W \mid D(A)$ is bounded with respect to the norm $\|\mathrm{P} \cdot\|$ on $D(A)=D(P)$. In the proof of the implication (ii) $\Rightarrow$ (iii) in Theorem 2.5 it was shown that $W$ is a bounded operator in $K$. By Theorem 1.2 it follows that 


$$
\mathrm{WD}\left(\mathrm{P}^{\alpha}\right) \leqq D\left(\mathrm{P}^{\alpha}\right) \quad(0 \leqq \alpha \leqq 1)
$$

Hence, we have proved that in the Krein space $K$ there exists a positive, bounded and boundedly invertible operator $W$ such that $W D\left(\mathrm{JP}^{\alpha}\right) \leqq D\left(\mathrm{JP}^{\alpha}\right) \quad(0 \leqq \alpha \leqq 1)$. Since the operator $\mathrm{JP} \mathrm{P}^{\mu}$ $(\mu \in(0,+\infty))$ is positive and boundedly invertible in the Krein space $K$ the implication (iii) $\Rightarrow$ (vii) in Theorem 2.5 yields $\infty \notin \mathrm{c}_{\mathrm{s}}\left(\mathrm{JP}^{\alpha}\right)$. Thus we have proved the "only if" part of the theorem for $\mu \in(0,1]$. For $\mu>1$ there exists a positive integer $m$ such that $\mu / 2^{\mathrm{m}}<1$. The operator $\mathrm{JP}^{2^{\mathrm{m}}}$ is positive, boundedly invertible and Lemma 2.8 implies $\infty \notin \mathrm{C}_{\mathrm{S}}\left(\mathrm{JP}^{2^{\mathrm{m}}}\right)$. Since $\mu / 2^{\mathrm{m}}<1$, we can apply the part of Theorem 2.9 which was proved already to the operator $\mathrm{JP}^{2^{\mathrm{m}}}$ and we get $\infty \notin c_{s}\left(J P^{\mu}\right),(\mu \in(1,+\infty))$. The "only if" part of the theorem is proved. In order to prove the "if" part of the theorem we apply the already proved "only if" part of the theorem to the operator $J P^{\mu}(\mu \in(0,+\infty)$ and $1 / \mu \in(0,+\infty)$. It follows that $\infty \notin \mathrm{C}_{\mathrm{s}}\left(J \mathrm{JP}^{\mu}\right)$ implies $\infty \notin \mathrm{c}_{\mathrm{s}}(\mathrm{JP})$. The theorem is proved.

\section{DEFINITIZABLE OPERATORS}

3.1. In this section we generalize the equivalence of the statements (ii), (iii), (iv) and (vii) in Theorem 2.5 to definitizable operators in the Krein space $K$ and give some applications of this result.

LEMMA 3.1. Let $A$ be a definitizable operator in the Krein space $(K,[\ldots])$. Then $\infty \notin \mathrm{C}_{\mathrm{S}}(\mathrm{A})$ if and only if $\infty \notin C_{s}(J(|J A|+I))$.

PROOF. Denote the spectral function of $A$ by $E$. Let $\Delta_{\infty}$ be such that $\mathbb{R} \backslash \Delta_{\infty}$ is a bounded interval containing all the finite critical points of $A$ and zero in its interior and put $K_{\infty}:=E\left(\Delta_{\infty}\right) K$. The restriction $A I_{K_{\infty}}$ is a boundedly 
invertible, positive operator in the $\operatorname{Krein}$ space $\left(K_{\infty},[\ldots]\right)$. Suppose $\infty \notin c_{S}(A)$ and let $J_{0}$ be a fundamental symmetry on $K$ commuting with $E\left(\Delta_{\infty}\right)$. We put $(x, y)_{0}:=\left[J_{0} x, y\right] \quad(x, y \in K)$ and $P_{0}:=J_{0} A$. Then $J_{0} J_{K_{\infty}}$ is a fundamental symmetry on $K_{\infty}$ and $P_{0}$ commutes with $E\left(\Delta_{\infty}\right)$. The operator $P_{0} I_{K_{\infty}}$ is positive and boundedly invertible in the Hilbert space $\left(K_{\infty},(\ldots)_{0}\right)$ and $P_{0} I_{K_{\infty}}=\left|P_{0}\right| I_{K_{\infty}}$. Now the following statements are equivalent:

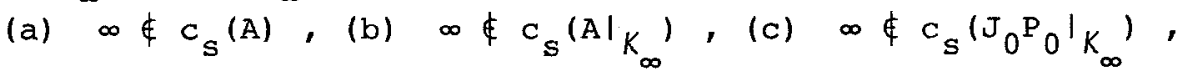
(d) $\infty \notin c_{s}\left(J_{0}\left|P_{0}\right| I_{K_{\infty}}\right),(e) \infty \notin c_{s}\left(J_{0}\left(\left|P_{0}\right|+I\right) I_{K_{\infty}}\right)$, (f) $\infty \notin c_{s}\left(J\left(\left|P_{0}\right|+I\right)\right),(g) \infty \notin c_{s}(J(|P|+I))$. The equivalences $(d) \Leftrightarrow(e)$ and $(f) \Leftrightarrow(g)$ are consequences of Corollary 2.6, the other equivalences are obvious. This completes the proof of the lemma.

The following theorem is a consequence of Lemma 3.1 , Remark 1.4 and Theorem 2.5 .

THEOREM 3.2. Let $A$ be a definitizable operator in the Krein space $(\mathrm{K},[.]$,$) . The following statements are equiva-$ Zent.

(i) Infinity is not a singular critical point of $\mathrm{A}$.

(ii) In the Krein space (K, [.,.]) there exists a positive, boundedly invertible operator $\mathrm{W}$ such that

$$
D(A) \subseteq D(W), W D(A) \subseteq D(A),
$$

and WI $D(A)$ is a bounded operator in $D(A) \wedge$.

(iii) In the Krein space (K,[.,.]) there exists a positive, bounded and boundedly invertible operator $W$ such that (3.1) holds.

(iv) In the Krein space (K, [.,.1) there exists a positive, boundedly invertible operator $\mathrm{W}$ such that

$$
D[J A] \subseteq D(W), W D[J A] \subseteq D[J A],
$$

and $\mathrm{WI}_{D[\mathrm{JA}]}$ is a bounded operator in $D[J A]^{\sim}$. 
COROLLARY 3.3. Let $\mathrm{A}$ and $\mathrm{B}$ be definitizable operators in the Krein space $K$ and suppose that $D(A)=D(B)$. Then $\infty \notin \mathrm{C}_{\mathbf{s}}(\mathrm{A})$ if and only if $\infty \notin \mathrm{c}_{\mathrm{s}}(\mathrm{B})$.

PROOF. This assertion is an easy consequence of the equivalence (iii) $\Leftrightarrow$ (i) in Theorem 3.2 and the assumption $D(A)=D(B)$.

REMARK 3.4. The preceding corollary yields the following perturbation results. Let $A$ and $B$ be operators in the Krein space $K$ such that $D(B) \supseteqq D(A) \quad(D(B) \supseteqq R(A)$, respectively) and such that the operators $A$ and $A+B(B A)$ are definitizable. Then $\infty \notin C_{S}(A)$ if and only if $\infty \notin c_{S}(A+B)$ $\left(\infty \notin c_{S}(B A)\right.$, respectively).

PROPOSITION 3.5. Let $A$ be a definitizable operator in the Krein space $K$. The following statements are equivalent. (i) Infinity is not a singular critical point of $\mathrm{A}$. (ii) In the Krein space $K$ there exists a positive, bounded and boundedly invertible operator $\mathrm{W}$ such that

$$
W D[J A] \subseteq D[J A]
$$

PROOF. Remark 1.4 implies $D[J A]=D\left(J(|J A|+I)^{1 / 2}\right)$. Consequently, the statement (ii) of this proposition coincides with the statement (iii) in Theorem 2.5 applied to the positive, boundedly invertible operator $\mathrm{J}(|\mathrm{JA}|+\mathrm{I})^{1 / 2}$. According to the equivalence (iii) $\Leftrightarrow$ (vii) in Theorem 2.5, in order to prove the proposition, it is sufficient to show that $\infty \notin c_{S}\left(J(|J A|+I)^{1 / 2}\right)$ if and only if $\infty \notin c_{S}(A)$. In order to prove this we note that Lema 2.8 yields $\infty \notin \mathrm{C}_{\mathrm{s}}\left(J(|J A|+I)^{1 / 2}\right)$ if and only if $\infty \notin \mathrm{C}_{\mathrm{s}}(\mathrm{J}(|\mathrm{JA}|+\mathrm{I}))$ and that Lemma 3.1 implies $\infty \notin \mathrm{c}_{\mathrm{s}}(\mathrm{J}(|\mathrm{JA}|+\mathrm{I}))$ if and only if $\infty \notin \mathrm{C}_{\mathrm{s}}(\mathrm{A})$. The proposition is proved.

COROLLARY 3.6. Let A and B be definitizable operators in the Krein space $K$. Suppose that $D$ [JA] $=D$ [JA]. Then $\infty \notin \mathrm{c}_{\mathbf{s}}(\mathrm{A})$ if and only if $\infty \notin \mathrm{c}_{\mathrm{s}}(\mathrm{B})$. PROOF. This assertion is an easy consequence of the 
equivalence in Proposition 3.5 and the assumption $D[J A]=D[J B]$. REMARK 3.7. Let $A$ be a definitizable operator in the Krein space $K$. We shall describe a situation in which the assumption (ii) of Proposition 3.5 is fulfilled. Suppose that there exist a fundamental symmetry $J$ and operators $X_{ \pm}, Y_{ \pm}$ defined on $K$ with the following properties:

$$
\begin{aligned}
& \text { (a) } \mathrm{X}_{ \pm} D[\mathrm{JA}] \subseteq D[J A], Y_{ \pm} D[J A] \subseteq D[J A], \\
& \text { (b) } \mathrm{X}_{ \pm} \text {and } Y_{ \pm} \text {are bounded in } K, \\
& \text { (c) } \mathrm{X}_{ \pm}{ }^{\prime} K_{ \pm}=I_{ \pm}, \mathrm{X}_{ \pm}\left(K_{\mp}\right) \subseteq K_{\mp}, \\
& \text { (d) } \mathrm{X}_{ \pm}=\mathrm{Y}_{ \pm}^{*} \mathrm{~J} .
\end{aligned}
$$

Here $I\left(I_{ \pm}\right.$, respectively) denotes the identity operator on $K\left(K_{ \pm}\right.$, respectively). Then the operator $W:=Y_{+} X_{+}+Y_{-} X_{-}$ has all the properties of the operator $W$ in Proposition 3.5 (ii). In order to prove this we only have to show that $w$ is boundedly invertible and positive in the krein space $K$. This follows from the relation

$$
\begin{aligned}
(x, x) & =\left(x_{+}, x_{+}\right)+\left(x_{-}, x_{-}\right)=\left(X_{+} x_{+}, X_{+} x_{+}\right)+\left(X_{-} x_{-}, X_{-} x_{-}\right) \\
& \leqq\left(X_{+} x_{,} Y_{+}^{* J x}\right)+\left(X_{-} x_{,} Y_{-}^{* J X}\right) \\
& =\left(J Y_{+} X_{+} x, x\right)+\left(J Y_{-} X_{-} x, x\right)=\left(J\left(Y_{+} X_{+}+Y_{-} X_{-}\right) x, x\right) \\
& =(J W x, x)=[W x, x]\left(x \in K, x_{ \pm}=P_{ \pm} X^{\prime}\right) .
\end{aligned}
$$

Operators $\mathrm{X}_{ \pm}, \mathrm{Y}_{ \pm}$with the above properties are constructed in [2] for a class of Sturm-Liouville operators with an indefinite weight function (see also [5]).

REMARK 3.8. Let $\mathrm{S}$ be a symmetric operator in the Hilbert space $(K,(.)$,$) which is bounded from below with a$ lower bound $\gamma$. Then the equality

$$
\|x\|_{s}^{2}:=(1-\gamma)\|x\|^{2}+(S x, x) \quad(x \in D(S))
$$

defines a norm on $D(S)$ (see $[23, p .122]$ ). Denote by $S_{F}$ the Friedrichs extension of $S$ and suppose that $J S_{F}$ is a definitizable operator in the Krein space $(K,[\ldots])$. Assume that 
there exists a positive, boundedly invertible operator $W$ such that

$$
D(S) \subseteq D(W), W D(S) \subseteq D(S)
$$

and such that $W I_{D(S)}$ is a bounded operator with respect to the norm $\|\cdot\|_{S} \cdot$ Then $\infty \notin C_{S}\left(J_{F}\right)$.

Indeed, the completion of $D(S)$ with respect to the norm $\|\cdot\|_{s}$ is evidently contained in $D(W)$ and invariant under $W$. This completion coincides with $D\left[S_{F}\right]$ ([14, Theorem 10]). The norm $\|\cdot\|_{S}$ can be extended onto $D\left[S_{F}\right]$ and for this extended norm $\|\cdot\|_{S}$ we have

$$
\|x\|_{S}=\left\|\left(S_{F}+(1-\gamma) I\right)^{1 / 2} x\right\| \quad\left(x \in D\left[S_{F}\right]\right),
$$

and $W I_{D}\left[S_{F}\right]$ is bounded with respect to this norm. Proposition 1.1 implies that the norm (3.2) generates the topology of $D\left[S_{F}\right]^{\sim}$. Hence, Theorem 3.2 yields $\infty \notin \mathrm{C}_{\mathrm{s}}\left(\mathrm{JS}_{\mathrm{F}}\right)$.

Denote by $S_{K}$ the Krein extension of $S$ (that is the soft extension in the terminology of [14]). Suppose that $\mathrm{JS}_{K}$ is a definitizable operator in the krein space $(K,[\ldots])$ and $\gamma>0$. If the operator $W$, in addition to the previous properties, is bounded and satisfies

$$
W R(J S) \cong R(J S)
$$

then $\infty \notin \mathrm{C}_{\mathrm{S}}\left(\mathrm{JS}_{\mathrm{K}}\right)$.

In order to prove this observe that by Theorem 14 in [14] we have

$$
D\left[\mathrm{~S}_{\mathrm{K}}\right]=D\left[\mathrm{~S}_{\mathrm{F}}\right]+\mathrm{N}_{\mathrm{O}}
$$

where $N_{0}$ is a kernel of the operator $S^{*}$. Furthermore $N_{0}$ is invariant under $W$. Indeed, for every $x \in D(S)$ there exists $x^{\prime} \in D(S)$ such that $W_{J S X}=J S x^{\prime}$. Hence, for $\varphi \in N_{0}$ we have 


$$
\begin{aligned}
\left(S^{*} W \varphi, x\right) & =(W \varphi, S x)=[W \varphi, J S x]=[\varphi, W J S x] \\
& =\left[\varphi, J S x^{\prime}\right]=\left(\varphi, S x^{\prime}\right)=\left(S^{*} \varphi, x^{\prime}\right)=0 .
\end{aligned}
$$

Since $\mathrm{x} \in D(\mathrm{~S})$ was arbitrary, and $D(S)$ is dense in $K$ we conclude that $W \varphi \in N_{0}$. We have seen that $D\left[S_{F}\right]$ is also invariant under $W$. Hence, $D\left[S_{K}\right]$ is invariant under the positive, bounded and boundedly invertible operator $W$ and Proposition 3.5 yields $\infty \notin \mathrm{C}_{\mathrm{S}}\left(\mathrm{JS}_{K}\right)$.

3.2. The following theorem is an extension of Theorem 2.9 for an operator with a nonempty resolvent set which is positive and selfadjoint in the Krein space.

THEOREM 3.9. Let $A$ be a positive, selfadjoint operator in the Krein space $K$ such that $\rho(A) \neq 0$, and put $\mathrm{P}=\mathrm{JA}$. If $\mu \in(0,+\infty)$ is such that $\rho(\mathrm{JP}) \neq \varnothing$ then $\infty \notin \mathrm{C}_{\mathrm{s}}(\mathrm{A})$ if and only if $\infty \notin \mathrm{C}_{\mathrm{s}}\left(J \mathrm{P}^{\mu}\right)$.

PROOF. Suppose $\rho\left(J P^{\mu}\right) \neq \varnothing(\mu \in(0,+\infty))$. In this case the operator $\mathrm{JP}^{\mu}$ is definitizable. The equality (1.3) implies that $D\left((P+I)^{\mu}\right)=D\left(P^{\mu}\right)$ and, according to Corollary $3.3, \infty \notin c_{s}\left(J P^{\mu}\right)$ if and only if $\infty \notin c_{s}\left(J(P+I)^{\mu}\right)$. Theorem 2.9 yields $\infty \notin \mathrm{C}_{\mathrm{s}}\left(\mathrm{J}(\mathrm{P}+\mathrm{I})^{\mu}\right)$ if and only if $\infty \notin \mathrm{C}_{\mathrm{S}}(J(P+I))$. Since $A$ is a definitizable operator Lemma 3.1 implies that $\infty \notin \mathrm{C}_{\mathrm{S}}(\mathrm{J}(\mathrm{P}+\mathrm{I}))$ if and only if $\infty \notin \mathrm{C}_{\mathrm{S}}(\mathrm{A})$. These equivalences prove the theorem.

According to Lemma 1.8 the condition $\rho\left(J \mathrm{P}^{\mu}\right) \neq \varnothing$ in Theorem 3.9 is satisfied for $\mu=2^{\mathrm{m}}, \mathrm{m}$ a positive integer. PROPOSITION 3.10. Let $A$ be a definitizable operator in the Krein space $K$ and put $\mathrm{P}=\mathrm{JA}$. Suppose that for some $\mathrm{m} \in\{\mathrm{n}, 1 /(2 \mathrm{n}+1): \mathrm{n}=1,2, \ldots\}$ the operator $\mathrm{JP}^{\mathrm{m}}$ is definitizable. Then $\infty \notin \mathrm{C}_{\mathrm{S}}(\mathrm{A})$ if and only if $\infty \notin \mathrm{C}_{\mathrm{S}}\left(\mathrm{JP}^{\mathrm{m}}\right)$.

PROOF. Lemma 3.1 implies that $\infty \notin \mathrm{C}_{\mathrm{S}}(\mathrm{A})$ if and only if $\infty \notin C_{S}(J(|P|+I))$. Theorem 2.9 yields $\infty \notin c_{s}(J(|P|+I))$ if and only if $\infty \notin \mathrm{C}_{S}\left(J(|P|+I)^{\mathrm{m}}\right)$. The equality (1.3) implies the first of the following equalities 


$$
D\left((|P|+I)^{m}\right)=D\left(|P|^{m}\right)=D\left(\left|P^{m}\right|\right)=D\left(P^{m}\right)=D\left(J P^{m}\right) .
$$

By assumption the operator $\mathrm{JP}^{\mathrm{m}}$ is definitizable, and Corollary 3.3 yields $\infty \notin \mathrm{c}_{S}\left(J(|P|+I)^{\mathrm{m}}\right)$ if and only if $\infty \notin \mathrm{c}_{\mathrm{s}}\left(J \mathrm{P}^{\mathrm{m}}\right)$. This sequence of equivalences proves the proposition.

PROPOSITION 3.11. Let $\mathrm{A}$ be a positive, seifadjoint operator in the Krein space $K$ such that $\rho(A) \neq \varnothing, 0 \notin \sigma_{\mathrm{p}}(\mathrm{A})$, and put $\mathrm{P}=\mathrm{JA}$. Suppose that $\rho\left(\mathrm{JP}^{\mu}\right) \neq \varnothing$ for some $\mu \in \mathbb{R} \backslash\{0\}$. Then $\mathbb{A}$ is fundamentally reducible if and only if $\mathrm{JP}^{\mu}$ is fundamentalzy reducible.

PROOF. The operators $\mathrm{A}, \mathrm{A}^{-1}, \mathrm{JP}^{\mu}$ and $\mathrm{JP}^{-\mu}$ are positive and selfadjoint in the krein space $K$ and these operators have nonempty resolvent sets. Therefore, only 0 and $\infty$ can be critical points of these operators. For $\mu>0$ Theorem 3.9 implies that $\infty \notin \mathrm{C}_{\mathrm{S}}(\mathrm{A})$ if and only if $\infty \notin \mathrm{C}_{\mathrm{S}}\left(\mathrm{JP} \mathrm{P}^{\mu}\right)$. Further, $0 \notin C_{S}(A)$ if and only if $\infty \notin C_{S}\left(A^{-1}\right)$. Because of $A^{-1}=J\left(\mathrm{JP}^{-1} \mathrm{~J}\right)$ and $\left(\mathrm{JP}^{-1} \mathrm{~J}\right)^{\mu}=\mathrm{JP}^{-\mu} \mathrm{J}$, according to Theorem 3.9, we have $\infty \notin c_{S}\left(A^{-1}\right)$ if and only if $\infty \notin c_{S}\left(P^{-\mu} J\right)$. Since $\left(F^{-\mu_{J}}\right)^{-1}=J P^{\mu}$, we conclude that $0 \notin c_{S}(A)$ if and only if $0 \notin \mathrm{C}_{\mathrm{S}}\left(\mathrm{JP}^{\mu}\right)$. Hence, for $\mu>0$, we have proved that $\mathrm{C}_{\mathbf{S}}(A)=\varnothing$ if and only if $c_{S}\left(J P^{\mu}\right)=\varnothing$. For $\mu<0$ the last equivalence follows from the equivalence: $c_{S}(A)=\varnothing \Leftrightarrow c_{S}\left(A^{-1}\right)=\varnothing$. For a positive, selfadjoint operator $B$ in the Krein space $K$ such that $\rho(B) \neq \varnothing$, the fundamental reducibility is equivalent to $c_{s}(B)=\varnothing$ (see $\left.[10]\right)$. The proposition is proved.

\section{ADDITIVE PERTURBATIONS}

In this section we show that the regularity of the critical point $\infty$ is "stable" under certain "additive" perturbations. This question was also considered in [22], [10], [11]. Here, however, we suppose that the perturbed operator has a definitizable extension. This allows us to weaken the conditions on the perturbing operator slightly. 
THEOREM 4.1. Let A be a definitizable, and let $\mathrm{B}$ be a symmetric operator in the Krein space $(K,[.]$,$) satisfying$ the following conditions:

(1) $D(B) \subseteq D(A)$ and $D(B)$ is a core of $|J A|^{1 / 2}$;

(2) $[A x, x] \geqq \gamma\|x\|^{2}(x \in D(A))$ for some $\gamma \in \mathbb{R}$

(that is JA is bounded from below);

(3) There exist $\alpha_{1}, \alpha_{2}, \beta_{1}, \beta_{2} \geqq 0, \beta_{1}<1$ such that

$-\alpha_{1}\|x\|^{2}-\beta_{1}(\| J A \mid x, x) \leqq[B x, x]$

$\leqq \alpha_{2}\|x\|^{2}+\beta_{2}(\| J A \mid x, x) \quad(x \in D(B))$.

Then the operator $\mathrm{J}(\mathrm{A}+\mathrm{B})$ is bounded from below. If its Friedrichs extension $\mathrm{S}$ has the property that $\mathrm{T}=\mathrm{JS}$ is definitizable, then $\infty \notin \mathrm{c}_{\mathbf{S}}(\mathrm{A})$ if and only if $\infty \notin \mathrm{c}_{\mathbf{S}}(\mathrm{T})$.

PROOF. It is easy to see that for $\alpha \leqq \gamma, \alpha \leqq 0$ the inequality

$$
(|J A| x, x) \leqq(J A x, x)-2 \alpha\|x\|^{2} \quad(x \in D(A))
$$

holds. The left inequality in (4.1) and (4.2) imply for $\mathrm{x} \in D(\mathrm{~B})$ and $\alpha \leqq \gamma, \alpha \leqq 0$ :

$$
\begin{aligned}
& (J(A+B) x, x) \geqq(J A x, x)-\beta_{1}(|J A| x, x)-\alpha_{1}\|x\|^{2} \\
& \geqq(J A x, x)-\beta_{1}(J A x, x)+2 \beta_{1} \alpha\|x\|^{2}-\alpha_{1}\|x\|^{2} \\
& \geqq\left(\left(1-\beta_{1}\right) \gamma+2 \beta_{1} \alpha-\alpha_{1}\right)\|x\|^{2} .
\end{aligned}
$$

Hence, the operator $J(A+B)$ is bounded from below in $(K,(.,)$.$) . Denote its lower bound by \delta$. Subtracting $\beta\|x\|^{2}, \beta<\delta$, from the first and the third term in (4.3), for $x \in D(B)$, we get

$$
\begin{aligned}
& (J(A+B) x, x)-\beta\|x\|^{2} \\
& \geqq\left(1-B_{1}\right)(J A x, x)+\left(2 \beta_{1} \alpha-\alpha_{1}-\beta\right)\|x\|^{2} .
\end{aligned}
$$

Further, (4.4) together with (4.2) and $1-\beta_{1}>0$, for $\beta<2 \alpha-\alpha_{1}$ and $\mathrm{x} \in D(B)$ implies that 


$$
\begin{aligned}
& (J(A+B) x, x)-\beta\|x\|^{2} \\
& \geqq\left(1-\beta_{1}\right)(\| J A \mid x, x)+\left(2 \alpha-\alpha_{1}-\beta\right)\|x\|^{2} \\
& =\left(1-\beta_{1}\right)\left\|\left(|J A|+\frac{2 \alpha-\alpha_{1}-\beta}{1-\beta_{1}} I\right)^{1 / 2} x\right\|^{2}
\end{aligned}
$$

The right inequality in (4.1), for $\beta^{\prime}<\delta, \beta^{\prime}<\alpha_{2}, x \in D(B)$, yields

$$
\begin{aligned}
0 & \leqq(J(A+B) x, x)-\beta^{\prime}\|x\|^{2} \\
& \leqq(J A x, x)+\beta_{2}(\| J A \mid x, x)+\alpha_{2}\|x\|^{2}-\beta^{\prime}\|x\|^{2} \\
& \leqq\left(1+\beta_{2}\right)(\| J A \mid x, x)+\left(\alpha_{2}-\beta^{\prime}\right)\|x\|^{2} \\
& =\left(1+\beta_{2}\right)\left\|\left(|J A|+\frac{\alpha_{2}-\beta^{\prime}}{1+\beta_{2}} I\right)^{1 / 2} x\right\|^{2} .
\end{aligned}
$$

The norms on the right hand sides in (4.5) and (4.6) are equivalent to the norm $\left\|(|J A|+I)^{1 / 2} \cdot\right\|$ on $D[J A]$. Since we have $D[J A]=D\left(|J A|^{1 / 2}\right)$, the assumption that $D(B)$ is a core of $|J A|^{1 / 2}$ implies that $D(B)$ is dense in $D\left(|J A|^{1 / 2}\right)$ in the graph norm. According to Proposition 1.1, the set $D(B)$ is dense in $D[J A]^{\sim}$. Now, the inequalities (4.5) and (4.6) imply that $D[J A]$ is the domain of the closure of the sesquilinear form $[(A+B) \cdot, \cdot]$ defined on $D(B) \quad($ see $[23, p .122])$. The Friedrichs extension $S$ of the operator $J(A+B)$ is bounded from below and the domains of the closures of the. sesquilinear forms $(J(A+B) \cdot, \cdot)$ and $(J S \cdot, \cdot)$ coincide. From the previous considerations and Remark 1.5 it follows that $D[J A]=D[J T]$. Since the operator $T$ is definitizable, corollary 3.6 implies that $\infty \notin c_{s}(A)$ if and only if $\infty \notin c_{s}(T)$. The theorem is proved.

\section{ACKNOWLEDGMENT}

This note was written during the author's stay at the Department of Mathematics of the Technical University, Dresden. The author is indebted to Professor Heinz Langer for calling his 
attention to these questions and for many valuable discussions. He also expresses his gratitude to Dr. Peter Jonas for many helpful suggestions and for continual interest in this work. The grant from German Democratic Republic and the support from the Research Council of SR Bosnia and Hercegovina, which made this stay in Dresden possible, are gratefully acknowledged.

\section{REFERENCES}

1. Bayasgalan, Ts.: On the fundamental reducibility of positive operators in spaces with indefinite metric. (Russian.) Studia Sci. Math. Hungar. 13 (1978), 143-150.

2. Beals, R.: Indefinite Sturm-Liouville problems and half-range completeness. J. Differential Equations, to appear.

3. Bognár, J.: Indefinite inner product spaces. Ergebnisse der Mathematik und ihrer Grenzgebiete 78, SpringerVerlag, Berlin / Heidelberg / New York, 1974.

4. Coddington, E.A., de Snoo, H.S.V.: Regular boundary value problems associated with pairs of ordinary differential expressions. Lecture Notes in Mathematics 858, Springer-Verlag, Berlin / Heidelberg / New York, 1981.

5. Curgus, B., Langer, H.: Spectral properties of selfadjoint ordinary differential operators with an indefinite weight function. Proceedings of the 1984 Workshop "Spectral theory of Sturm-Liouville differential operators". ANL-84-73, Argonne National Laboratory, Argonne, Illinois, 1984, 73-80.

6. Hess, P.: Zur Theorie der linearen Operatoren eines $\mathrm{J}$-Raumes. Operatoren, die von kanonischen Zerlegungen reduziert werden. Math. Z. 106 (1968), 88-96.

7. Jonas, P.: úber die Erhaltung der Stabilität J-positiver Operatoren bei J-positiven und J-negativen störungen. Math. Nachr. 65 (1975), 211-218. 
8. Jonas, P.: Relatively compact perturbations of uniformly J-positive operators. Akademie der Wissenschaften der DDR, Zentralinstitut für Mathematik und Mechanik, Preprint P-15/80, Berlin, 1980 .

9. Jonas, P.: On the functional calculus and the spectral function for definitizable operators in Krein spaces. Beiträge Anal. 16 (1981), 121-135.

10. Jonas, P.: Compact perturbations of definitizable operators. II. J. Operator Theory 8 (1982), 3-18. 11. Jonas, P.: Regularity criteria for critical points of definitizable operators. Spectral theory of linear operators and related topics, 8th international conference on operator theory, Timişoara and Herculane (Romania), June 6-16, 1983. Operator Theory: Advances and Applications 14, Birkhäuser Verlag, Basel / Boston / Stuttgart, 1984, 179-195.

12. Kaper, H.G., Kwong, M.K., Lekkerkerker, C.G., Zettl, A.: Full- and partial-range eigenfunction expansions for Sturm-Liouville problems with indefinite weights. Proc. Roy. Soc. Edinburgh Sect. A 98 (1984), 69-88.

13. Kato, T.: Perturbation theory for linear operators. Die Grundlehren der mathematischen wissenschaften in Einzeldarstellungen 132, Springer-Verlag, Berlin / Heidelberg / New York, 1966.

14. Krein, M.G.: The theory of self-adjoint extensions of semi-bounded Hermitian transformations and its applications. I - II. (Russian.) Mat. Sb. (N.S.) 20 (62) (1947), 431-495, and 21 (63) (1947), 365-404.

15. Krein, M.G.: Completely continuous linear operators in function spaces with two norms. (Ukrainian.) Akad. Nauk Ukrain. RSR. Zbirnik Prac' Inst. Mat. 9 (1947), 104-129.

16. Krein, S.G.: Linear differential equations in Banach space. (Russian.) Izdatel'stvo "Nauka", Moscow / Leningrad, 1967. 
17. Langer, H.: Maximal dual pairs of invariant subspaces of J-selfadjoint operators. (Russian.) Mat. Zametki 7 (1970), 443-447.

18. Langer, H.: Verallgemeinerte Resolventen eines J-nichtnegativen operators mit endlichem Defekt. J. Funct. Anal. 8 (1971), 287-320.

19. Langer, H.: Spectral functions of definitizable operators in Krein spaces. Functional analysis, Proceedings of a conference held at Dubrovnik, Yugoslavia, November 2-14, 1981. Lecture Notes in Mathematics 948, Springer-Verlag, Berlin / Heidelberg / New York, 1982, $1-46$.

20. Lax, P.D.: Symmetrizable linear transformations. Comm. Pure Appl. Math. 7 (1954), 633-647.

21. Reid, W.T.: Symmetrizable completely continuous linear transformations in Hilbert space. Duke Math. J. 18 (1951), 41-56.

22. Veselić, K.: On spectral properties of a class of J-selfadjoint operators. I. Glasnik Mat. Ser. III 7 (1972), 229-248.

23. Weidmann, J.: Linear operators in Hilbert spaces. Graduate Texts of Mathematics 68, Springer-Verlag, New York / Heidelberg / Berlin, 1980.

Prirodno-matematicki fakultet Univerziteta u Sarajevu

Vojvode Putnika 43

YU 71000 Sarajevo

Yugoslavia 